Cite this: Soft Matter, 2013, 9, 6752

Received 11th January 2013

Accepted 27th February 2013

DOI: $10.1039 / \mathrm{c} 3 \mathrm{sm} 00131 \mathrm{~h}$

www.rsc.org/softmatter

\section{Site-specific, covalent incorporation of Tus, a DNA-binding protein, on ionic-complementary self-assembling peptide hydrogels using transpeptidase Sortase $A$ as a conjugation toolt\#}

\author{
Susanna Piluso, ${ }^{a}$ Heather C. Cassell, ${ }^{\text {b }}$ Jonathan L. Gibbons, ${ }^{c}{ }^{\text {Thomas E. Waller, }}{ }^{a}$ \\ Nick J. Plant, ${ }^{b}$ Aline F. Miller ${ }^{c}$ and Gabriel Cavalli*a
}

\begin{abstract}
The site-specific conjugation of DNA-binding protein (Tus) to selfassembling peptide FEFEFKFKK was demonstrated. Rheology studies and TEM of the corresponding hydrogels (including PNIPAAm-containing systems) showed no significant variation in properties and hydrogel morphology compared to FEFEFKFKK. Critically, we demonstrate that Tus is accessible within the gel network displaying DNA-binding properties.
\end{abstract}

\section{Introduction}

The self-assembly of ionic complementary octapeptides into $\beta$-sheet rich fibrous hydrogels is a well-studied phenomenon where the fibre structure, topology of the resulting network and its mechanical behaviour can be tailored by varying the peptide concentration and/or the ionic strength of the medium..$^{1-3}$ Their conjugation to stimuli-responsive polymers such as poly( $N$-isopropylacrylamide) (PNIPAAm) and the behaviour of the corresponding hydrogels has also been investigated. ${ }^{4,5}$ Hydrogels based on these octapeptides have potential for a wide range of biomedical applications. ${ }^{6,7}$ Due to the characteristics of such hydrogels, they have been found to mimic biological extracellular matrices, being ideal candidates for scaffolds for cell growth and tissue engineering. ${ }^{6,7}$ The covalent conjugation of functional proteins to these peptide scaffolds, thus incorporating biological functionality to the corresponding hydrogels, has not yet been reported. This appears to be the next logical step to expand the biofunctionality of these materials, and hence widen their potential in biomedical applications.

\footnotetext{
${ }^{a}$ Department of Chemistry, University of Surrey, Guildford, GU2 7XH, UK. E-mail: g.cavalli@surrey.ac.uk; Tel: +44 (0)1483686837

${ }^{b}$ Department of Biochemistry and Physiology, University of Surrey, Guildford, GU2 7XH, UK. E-mail: n.plant@surrey.ac.uk; Tel: +44 (0)1483686412

'Manchester Institute of Biotechnology, School of Chemical Engineering \& Analytical Science, University of Manchester, 131 Princess Street, Manchester, M1 7DN, UK. E-mail: aline.miller@manchester.ac.uk; Tel: +44 (0)161 3065781

$\dagger$ Dedicated to the memory of Joachim H. G. Steinke.

‡ Electronic supplementary information (ESI) available: Further experimental data. See DOI: 10.1039/c3sm00131h
}

Previously, we demonstrated the site-specific conjugation of C-terminus modified proteins to polymeric supports bearing diglycine moieties by means of $S$. aureus transpeptidase Sortase A. ${ }^{8}$ This system simply requires the presence of a C-terminus LPETGG sequence in the protein (where L: leucine, P: proline, E: glutamic acid, T: threonine and G: glycine), which can be readily incorporated during protein expression using routine molecular biology techniques. We have previously demonstrated that biological functionality is tolerant to this bioconjugation methodology when performed on polymer supports through the use of a DNA-binding protein, Tus, which recognises a 21 base pair specific DNA sequence called Ter site. ${ }^{8}$ The site-specific nature, relative to both conjugate partners, of the attachment using Sortase A appeared to us perfectly suited to peptide systems containing a large density of amino- and carboxylic moieties, such as those normally used in traditional bioconjugation approaches. Herein, we disclose the conjugation of Tus protein to a self-assembling ionic complementary peptide, FEFEFKFKK (where F: phenylalanine, E: glutamic acid and K: lysine) using Sortase A, as a proof of concept. We selected this peptide as it has a charge of +1 at physiological $\mathrm{pH}$, which renders it more soluble than its partner octapeptideFEFEFKFK-at $\mathrm{pH} 7 .^{6}$

Increasingly, a large number of publications in this area incorporate stimuli-responsive polymers, such as PNIPAAm. ${ }^{4,5}$ Therefore, investigating the effect of covalent protein attachment to FEFEFKFKK on the co-assembly with PNIPAAm-containing octapeptides, becomes as relevant as studying its co-assembly to naked FEFEFKFKK. Conversely, the effect of stimuli responsive polymer-containing peptides on the retention or otherwise of protein function, is also critical if this approach is to be extended in this field. In particular, this is critical since the presence of PNIPAAm has been reported to affect protein folding, and hence show a profound impact on protein function. ${ }^{9}$ Thus, this work addresses the following hypotheses: (1) Sortase A bioconjugation is applicable to incorporate functional proteins within peptide hydrogel systems; (2) protein containing-peptides can co-assemble with 
both polymer-containing- and non-functionalised peptides in a robust manner; (3) stimuli-responsive polymer systems are compatible with functional proteins when incorporated within peptide hydrogels.

\section{Results and discussion}

Peptides FEFEFKFKK (P1), peptide-initiator Br-FEFEFKFKK (P2) and GGFEFEFKFKK (P3), (where G is glycine) were synthesised following standard Fmoc peptide synthesis protocols. ${ }^{10}$ Peptides P2 and P3 were obtained from P1 by coupling the last amino acid, prior to acidic cleavage from the resin, with 2-bromoisobutyric acid, or Fmoc-glycylglycine followed by conventional piperidine Fmoc-group removal, respectively. The peptides were recovered by precipitation in cold diethyl ether, centrifugation and lyophilisation for three days. The synthesis yield was $64 \%$ for $\mathbf{P 1}, 98 \%$ for $\mathbf{P 2}$ and $45 \%$ for $\mathbf{P 3}$. The product identity was confirmed by LC-MS and ${ }^{1} \mathrm{H}-\mathrm{NMR}$. The purity of these peptides was at least 95\%; therefore they were used without further purification. GGFEFEFKFKK was used as the functional peptide for the incorporation of Tus protein using the Sortase A methodology of conjugation. ${ }^{8}$

A PNIPAAm-FEFEFKFKK conjugate (P4) was prepared by single-electron-transfer mediated living radical polymerisation (SET-LRP) using $\mathbf{P 2}$ as initiator and following conditions reported by Albertsson and Edlund. ${ }^{\mathbf{1 1}}$ The polymer-peptide conjugate P4 identity was confirmed by ${ }^{1} \mathrm{H}-\mathrm{NMR}$, and the results were consistent with those reported for a similar conjugate by Miller et al. ${ }^{4}$ As reported before, characterisation of $\mathbf{P 4}$ by GPC is complex due to the aggregating nature of the peptide; however, end group analysis in NMR data was used to calculate the molecular weight of the conjugate $\left(M_{\mathrm{n}}: 2600 \mathrm{~g} \mathrm{~mol}^{-1}\right)$. The LCST of $\mathbf{P 4}$, determined by turbidimetry, was found to be $31^{\circ} \mathrm{C}$, which was in line with similar PNIPAAm-peptide systems of the same molecular weight, making the behaviour of $\mathbf{P 4}$ comparable to that reported elsewhere. ${ }^{4}$ Tus protein was expressed incorporating the LPETGG C-terminus sequence for Sortase A recognition using plasmids transformed into Rosetta-Gami B(DE3) pLysS cells. A His ${ }_{6}$ tag was also incorporated to facilitate protein purification using affinity chromatography. Sortase A was similarly expressed and purified. Tus protein was conjugated to P3 following our previously reported Sortase A methodology. ${ }^{8}$ The Tus-GGFEFEFFKFKK conjugate (P5) was analysed by gelelectrophoresis, and purified by membrane centrifugation and affinity chromatography (Scheme 1 ).

Mixed hydrogels were prepared by incubating Tus-conjugate P5 and/or PNIPAAm-conjugate P4 with P1 in distilled water. Previously, these peptide-peptide-conjugate composite samples have been mixed at temperatures $>60^{\circ} \mathrm{C}$ to ensure homogeneity across the self-assembled samples. ${ }^{1-5}$ However, to avoid any unwanted protein denaturation peptides were mixed herein at $40{ }^{\circ} \mathrm{C}$ for $30 \mathrm{~min}$ and incubated overnight at room temperature (r.t.). In any case, for pure peptide, P1, oscillatory rheology measurements of the gel showed no significant difference between the two different gel preparation methods (see ESI $\neq$ ). In all cases, P5 was incorporated at 5\% (w/w) and $\mathbf{P 4}$ at $10 \%$ $(\mathrm{w} / \mathrm{w})$ with the remainder being $\mathbf{P 1}$, at a total concentration of
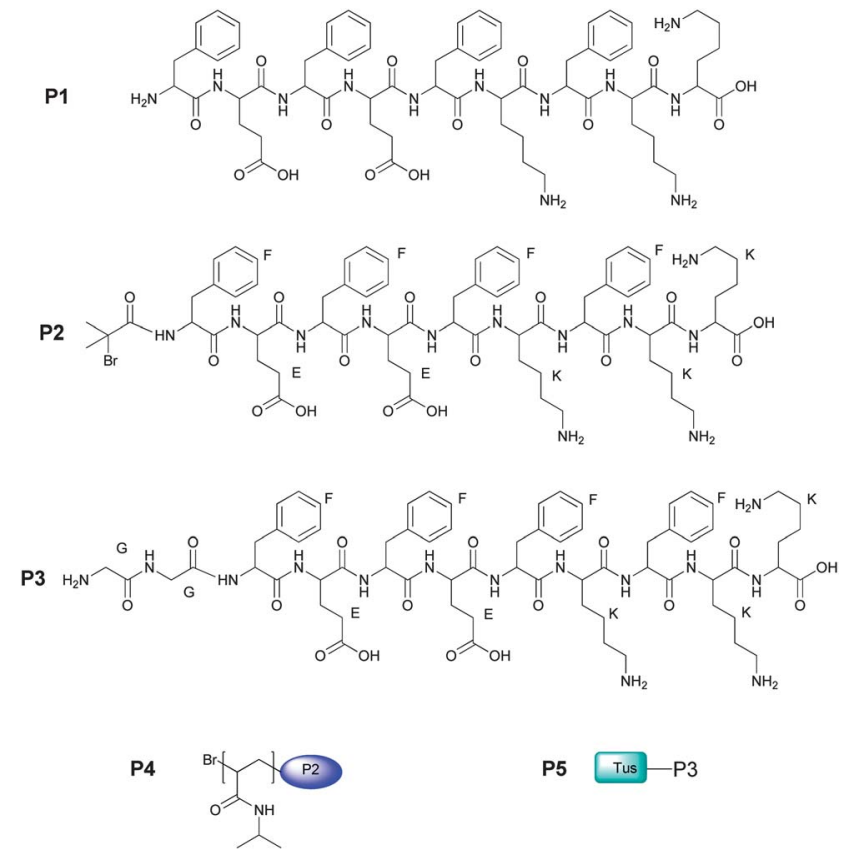

P5

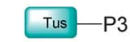

Scheme 1 Peptide structures and their conjugates.

$20 \mathrm{mg} \mathrm{ml}^{-1}$ (overall mass/vol). Thus, we prepared hydrogels from P1 only (PG1), P5/P1 (5/95\%, w/w) (PG2), P4/P1 (10/90\%, w/w) (PG3), P5/P4/P1 (5/10/85\%, w/w) (PG4) (Table 1). Given the molecular weight of the corresponding unimers, this guarantees a molar ratio of ca. 550 P1/P5, 20 P1/P4 and 30 P4/P5. Hydrogel formation was observed in all cases using the standard tilt test tube method. To confirm gel formation, the mechanical properties of PG1-4 were investigated using oscillatory rheology. The elastic $\left(G^{\prime}\right)$ and viscous $\left(G^{\prime \prime}\right)$ moduli were measured as a function of frequency $(0.1-10 \mathrm{~Hz})$ at $20,30,40$ and $50{ }^{\circ} \mathrm{C}$.

For all samples the elastic modulus was higher than the viscous modulus and no crossover point between $G^{\prime}$ and $G^{\prime \prime}$ was observed within the range explored (see ESI $\neq$ for further data). All values of $G^{\prime}$ were rather low, which indicates the presence of a weak gel network. This has the advantage of facilitating diffusion of DNA and promoting the accessibility of Tus for DNA binding, thereby reducing the potential of obtaining false negative results. Future work will involve increasing the concentration of peptide used, and the ionic strength of the

Table 1 Hydrogel composition

\begin{tabular}{lccr}
\hline & \multicolumn{2}{l}{ Hydrogel composition $\%(w / w)^{a}$} & P5 \\
\cline { 2 - 4 } & P1 & P4 & \\
\hline PG1 & 100 & & 5 \\
PG2 & 95 & 10 & \\
PG3 & 90 & 10 & 5
\end{tabular}

${ }^{a}$ Weight $\%$ of overall composition (peptide molar ratio shown in text). 


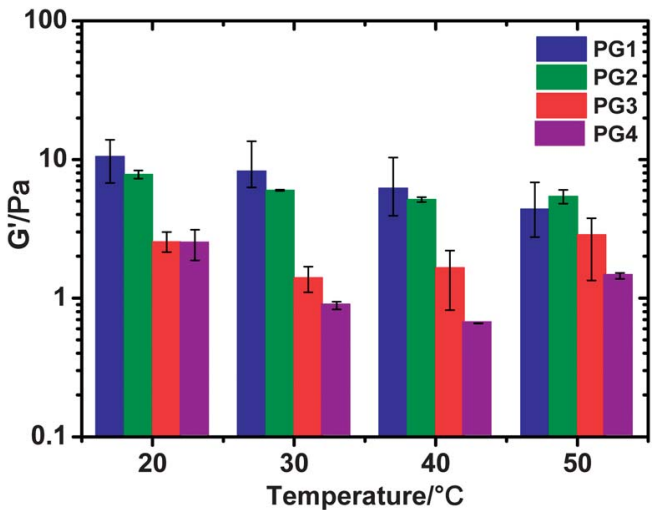

Fig. 1 The elastic modulus $\left(G^{\prime}\right)$ for all peptide hydrogels PG1-4 at different temperatures (measured at $1 \mathrm{~Hz}$ ). These values have been normalised to peptide content.

media, to manipulate the mechanical properties of the gels and explore its influence on the activity of the protein. This will be the subject of a forthcoming article. ${ }^{4}$ For the purpose of this proof-of-principle work, we proceeded with our PG1-4 samples.

Fig. 1 summarises the variation of $G^{\prime}$ as a function of temperature for each of the four samples. It is clear that for the PG1 and PG2 samples at $20^{\circ} \mathrm{C}, G^{\prime}$ remains roughly constant at $\sim 10 \mathrm{~Pa}$. This indicates that the peptide component from the peptide-Tus conjugate must be self-assembling into the $\beta$-sheet fibres and contributing to network formation. This will essentially leave the Tus component tethered to the surface of the fibre, although further work in the future will be necessary to confirm this point. Interestingly, attempts to self assemble FEFEFKFKK (P1) in the presence of unconjugated Tus protein resulted in no gelation observed. Although we currently have no explanation for this observation, this strongly suggests that covalent attachment is vital for co-assembly, indirectly supporting the idea of the protein being tethered to the fibre, in line with the rheology data. For the samples containing PNIPAAm, $G^{\prime}$ values are slightly lower at $\sim 2 \mathrm{~Pa}$, but interestingly this does not change upon addition of the peptide-Tus conjugate. As the temperature increases $G^{\prime}$ does not vary within experimental error for PG1 and PG2, indicating there is no change in sample morphology. For the two samples containing the thermoresponsive PNIPAAm, a slight decrease in $G^{\prime}$ is observed at $30{ }^{\circ} \mathrm{C}$, which then increases slightly as the temperature increases from 30 to $50{ }^{\circ} \mathrm{C}$. Similar trends were observed for both samples suggesting that the presence of Tus does not interfere with the behaviour of the polymer. The temperature range of such changes correlates well with the temperature where PNIPAAm is known to undergo its lower critical solution temperature (LCST). ${ }^{4,5}$ Parallel macroscopic experiments show that these two samples remain clear, with no precipitation or aggregation of the PNIPAAm chains being evident in the range $25-40{ }^{\circ} \mathrm{C}$ while a solution of PNIPAAm-conjugate (P4) exhibits a clear precipitate due to the polymer LCST at $T>31{ }^{\circ} \mathrm{C}$. This further suggests that the peptide component of the conjugate is participating to fibre formation as the tethered PNIPAAm is not able to form aggregates, despite going through its coil to globule transition.
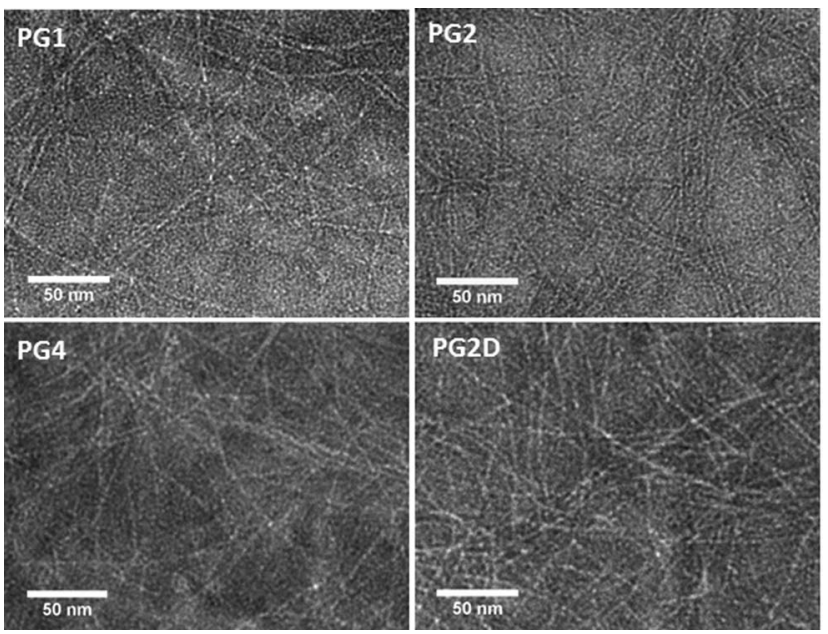

Fig. 2 TEM images of PG1 (top left), PG2 (top right), PG4 (bottom left) and PG2 after incubation with 100\% FAM-DNA (bottom right).

Instead the polymer chains will simply collapse along the surface of the fibre, homogeneously throughout the gel.

Fibre formation within each gel system was confirmed using TEM (Fig. 2). These show that there is no significant variation in fibre morphology for the conjugate containing gels (PG2-4) in comparison to the morphology observed for the peptide only hydrogel, PG1. The mean fibre diameter measured from TEM micrographs for all hydrogels was $\sim 2.7 \mathrm{~nm} \pm 0.3$, which correlate with those reported in the literature. ${ }^{2}$

Having demonstrated that protein-containing peptides are capable of forming hydrogels with robustness comparable to that of the polymer-containing and non-functionalised peptides, we investigated whether Tus was still accessible within the hydrogel network. To this end, we treated the Tus-containing PG2 with mixtures of Ter DNA labelled with fluorescein (FAM-Ter-DNA, $\mathbf{D N A}_{\mathbf{S}}$, where S stands for "specific Ter sequence") and a non-Ter related 21 base pair DNA sequence labelled with Cy5 (Cy5-nonTer-DNA, $\mathbf{D N A}_{\mathbf{N S}}$, where NS stands for "non Ter sequence") in binding buffer ( $50 \mathrm{mM}$ Tris-HCl, $250 \mathrm{mM} \mathrm{KCl}, 0.1 \mathrm{mM}$ EDTA, $0.1 \mathrm{mM}$ DTT, pH 9) overnight at r.t. The total DNA concentration $\left(\mathbf{D N A}_{\mathbf{S}}+\mathbf{D N A}_{\mathbf{N S}}\right)$ was $10 \mu \mathrm{M}$ for all samples in different proportions of $\mathbf{D N A}_{\mathbf{S}} / \mathbf{D N A}_{\mathbf{N S}}$, followed by washings with incubating buffer and measuring fluorescence of the gels on a green and red channel using a plate reader (Fig. 3). ${ }^{8}$ This way, we were able to discriminate between non-specific and specific binding on the gel at different Ter-DNA ratios $v s$. overall DNA concentration. The fluorescence data suggests a concentration dependent behaviour; although it is clear that under these conditions the hydrogel Tusfunctionality was near saturation levels even at the lowest DNA concentration tested (Fig. 3). Although more data would be required to investigate this behaviour, as well as quantifying the extent of functionality retention $v s$. incorporated protein, we were pleased for the confirmation that Tus protein remained functional and accessible to its cognate DNA sequence (Ter, $\left.\mathbf{D N A}_{\mathbf{s}}\right)$ on the hydrogel. Most importantly, the levels of non-specific binding were negligible, consistent with the low affinity of Tus for nonspecific DNA in $250 \mathrm{mM} \mathrm{KCl} .^{12}$ Further interrogation of PG2 

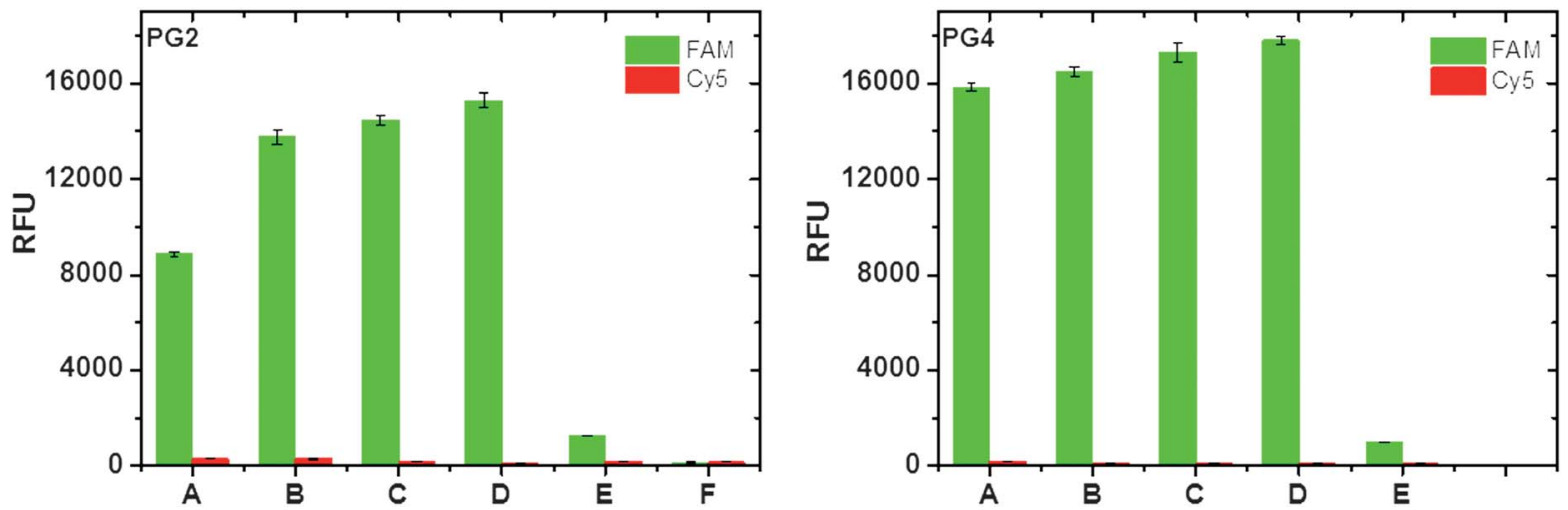

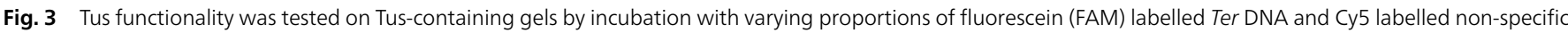

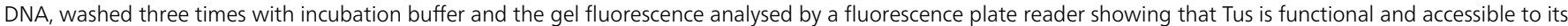

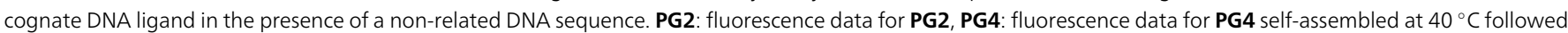

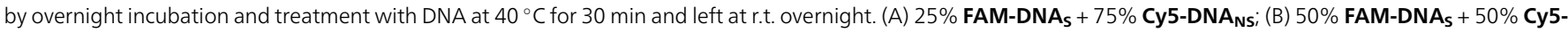

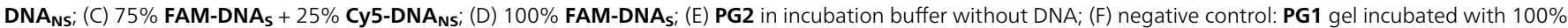
FAM-DNAs

samples by confocal fluorescence microscopy confirmed these observations (Fig. 4). As expected, an uneven distribution of Tusrelated green fluorescence upon FAM-labelled $\mathbf{D N A}_{\mathbf{S}}$ binding was observed in the hydrogel.

We proceeded further to similarly interrogate the PNIPAAm/ Tus containing gel (PG4). To test if the polymer phase transition had an effect on protein functionality PG4 was self-assembled by heating at $40{ }^{\circ} \mathrm{C}$ for $30 \mathrm{~min}$ ( $T>$ LCST for PNIPAAm), then left to cool down to r.t. overnight. Afterwards, PG4 was treated with different mixtures of $\mathbf{D N A}_{\mathbf{S}}+\mathbf{D N A}_{\mathbf{N s}}$ as explained above, again heating at $40{ }^{\circ} \mathrm{C}$ for $30 \mathrm{~min}$ followed by further overnight incubation at r.t. with DNA. We reasoned that the ratio of P4/P5 would at least ensure interaction prior to gelation, which required overnight incubation. The fluorescence levels after washing with incubating buffer were similar to PG2 (Fig. 3) also displaying negligible levels of non-specific binding. These results confirmed that Tus was tolerant to the co-assembly with PNIPAAm-containing peptides.

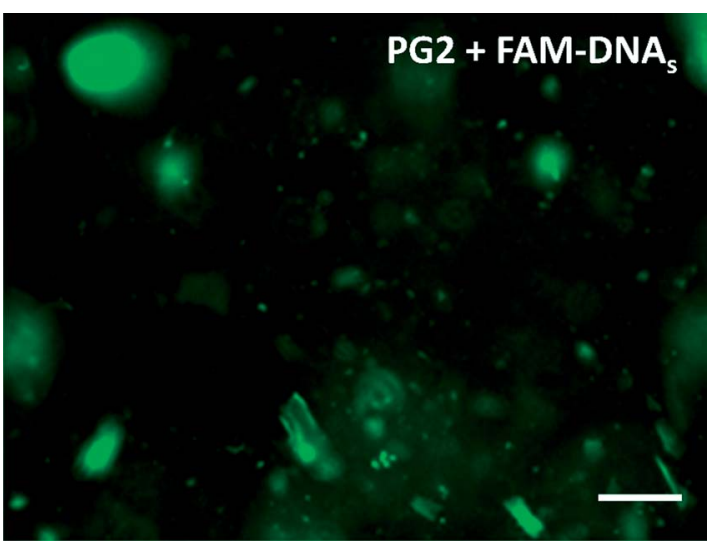

Fig. 4 Fluorescence microscopy image using FITC green fluorescence filter of gel PG2 treated with FAM-DNA showing specific binding of Ter-DNA to the Tuscontaining hydrogel. Magnification 100× (scale bar: $100 \mu \mathrm{m})$.

\section{Conclusions}

We have successfully demonstrated the site-specific covalent attachment of a functional protein (Tus) onto an ioniccomplementary self-assembling peptide hydrogel using Sortase A, as well as its ability to co-assemble both with unfunctionalised and unfunctionalised/peptide-polymer conjugated mixtures. Critically, the investigated protein remained functional and accessible in the final hydrogel, although the extent of this retention of functionality requires further investigation. We expect, in the future, to extend this principle to investigate other protein systems towards incorporating protein functionality on peptide hydrogels.

\section{Acknowledgements}

The authors would like to thank the British Heart Foundation for funding for SP and HCC (grant NH/11/7/29032), The School of Chemical Engineering \& Analytical Science (University of Manchester) for providing JLG with DTA funding, the Department of Chemistry (University of Surrey) for providing TEW with DTA funding and Cameron Neylon for kindly supplying plasmids for Sortase A and Tus protein, as well as invaluable discussions over this manuscript.

\section{References}

1 A. Mohammed, A. F. Miller and A. Saiani, Macromol. Symp., 2007, 258, 88.

2 S. Boothroyd, A. Saiani and A. F. Miller, Macromol. Symp., 2008, 273, 139.

3 A. Saiani, A. Mohammed, H. Frielinghaus, R. Collins, N. Hodson, C. M. Kielty, M. J. Sherrattf and A. F. Miller, Soft Matter, 2009, 5, 193.

4 A. Maslovskis, N. Tirelli, A. Saiani and A. F. Miller, Soft Matter, 2011, 7, 6025. 
5 F. Stoica, C. Alexander, N. Tirelli, A. F. Miller and A. Saiani, Chem. Commun., 2008, 4433.

6 D. Roberts, C. Rochas, A. Saiani and A. F. Miller, Langmuir, 2012, 28, 16196.

7 A. Mujeeb, A. F. Miller, A. Saiani and J. E. Gough, Acta Biomater., 2013, 9, 4609.

8 L. Chan, H. F. Cross, J. K. She, G. Cavalli, H. F. P. Martins and C. Neylon, PLoS One, 2007, 2, e1164.
9 H. Jones, J. Dalmaris, M. Wright, J. H. G. Steinke and A. D. Miller, Org. Biomol. Chem., 2006, 4, 2568.

10 Fmoc Solid Phase Peptide Synthesis, ed. W. Chan and P. White, Oxford University Press, New York, 2000.

11 U. Edlund and A.-C. Albertsson, J. Polym. Sci., Part A: Polym. Chem., 2012, 50, 2650.

12 C. Neylon, S. E. Brown, A. V. Kralicek, C. S. Miles, C. A. Love and N. E. Dixon, Biochemistry, 2000, 39, 11989. 